\title{
Missing Dipole Excitation Strength below the Particle Threshold
}

\author{
A.P. Tonchev ${ }^{a}$, C. Angell ${ }^{b}$, M. Boswell ${ }^{b}$, C.R. Howell ${ }^{a}$, H.J. Karwowski ${ }^{b}$, \\ J.H. Kelley ${ }^{c}$, W. Tornow ${ }^{a}$, N. Tsoneva ${ }^{d}$ \\ ${ }^{a}$ Duke University and TUNL, Department of Physics, Box 90308, Durham, NC 27708-0308, USA \\ ${ }^{b}$ University of North Carolina, Department of Physics and Astronomy, Chapel Hill, NC \\ 27599-3255, USA \\ ${ }^{c}$ North Carolina State University and TUNL, Department of Physics, Box 8202, Raleigh, NC \\ 27695-8202, USA \\ ${ }^{d}$ Institute of Theoretical Physics, University of Giessen, Heinrich-Buff-Ring 16, D-35392, \\ Giessen, Germany \\ E-mail: tonchevetunl.duke.edu
}

\begin{abstract}
High sensitivity studies of $\mathrm{E} 1$ and $\mathrm{M} 1$ excitations from the ${ }^{138} \mathrm{Ba}\left(\gamma, \gamma^{\prime}\right)$ reaction at energies close to the neutron emission threshold have been performed. The electric dipole character of the "pygmy" mode was experimentally verified for excitations from 5.5-8.5 MeV. Missing dipole strength has been reveal in our photon-scattering measurements.
\end{abstract}

Workshop on Photon Strength Functions and Related Topics

June 17-20 2007

Prague, Czech Republic 


\section{Introduction}

Wide variety of nuclear structure phenomena has been investigated with the nuclear resonance fluorescence (NRF) technique [1, 2, 3, 4]. Experiments probing two-phonon excitations of even-even nuclei near closed shells revealed large magnetic dipole strengths. The corresponding excitations have been associated with scissors-like oscillations of the deformed proton density distribution against the neutron distribution, and this excitation mode was, accordingly, called the scissors mode [5]. Large electric dipole transitions to the ground states have been observed in the spherical nuclei with $\mathrm{Z}=50$ and $\mathrm{N}=82$. They are assumed to arise from the coupling of quadrupole and octupole vibrational modes of the nucleus $[6,7]$. In the heavier nuclei a resonance like concentration of E1 strength has been identified below the neutron separation energy in semi-magic $\mathrm{N}=82$ isotones [8]. From the recent photon scattering experiments [9] a large data set on E1 excitations in $\mathrm{N}=82$ nuclei has been compiled. For example, in ${ }^{138} \mathrm{Ba}$ alone more than 70 dipole states have been observed in the energy range from 4 to $8.5 \mathrm{MeV}$. For the vast majority of the observed transitions only one decay branch to the ground state has been measured. However, this data set is still incomplete since the polarization sensitivity of the used "Compton polarimeters" [9] is very low above $4 \mathrm{MeV}$, making parity assignment impossible.

During the year of 2006, the experimental activities at the High-Intensity Gamma-ray Source (HI $\gamma \mathrm{S}$ ) have focused on investigation of this collective mode, commonly referred to as a "pygmy" dipole resonance (PDR), which is observed as a clustering of states close to the neutron threshold. Theoretical calculations indicate a correlation between the observed total $\mathrm{B}(\mathrm{E} 1)$ strength of the PDR and the neutron-to-proton ratio N/Z [10]. Although carrying only a small fraction of the full dipole strength, these states are of particular interest because they are interpreted as a motion of the neutron skin against the isotropic symmetric core. The observation of this collective dipole mode near the neutron threshold might have important astrophysical implications. For example, it has been shown that the nucleosynthesis of certain neutron deficient nuclei, so called $p$-nuclei, is strongly influenced by PDR structures [11]. The purpose of this paper is determine first, the character of "pygmy" mode of excitation. Is it E1 or M1 mode of excitation? Second, what is the decay pattern of these collective states below the particle separation energy? Third, what are the strength, energy distribution, and nature of these collective phenomena? To answer these questions we focus our experimental activity on the ${ }^{138} \mathrm{Ba}\left(\gamma, \gamma^{\prime}\right)$ reaction below the neutron separation energy.

\section{Experimental Considerations}

The HI $\gamma \mathrm{S}$ facility is used to produce high-intensity and nearly monoenergetic photon beams by intracavity Compton backscattering [12]. In $\gamma$-ray production mode two electron bunches stored in the Duke storage ring are synchronized so that the lased photons from one electron bunch are reflected by the downstream mirror, and then collide head on with the second electron bunch. The backscattered photons are collimated by a cylindrical lead collimator, with a diameter of 1.27 $\mathrm{cm}$, located $60 \mathrm{~m}$ downstream of the collision point. In addition, the $\gamma$-rays are highly polarized resulting from the Compton scattering process of $\approx 100 \%$ polarized photons.

The NRF method is used at HI $\gamma \mathrm{S}$ to study low-multipolarity ground state transitions (i.e., E1 and M1) with large partial widths. Due to the low detection limit the NRF technique represents 




Figure 1: Quartet of 60\% HPGe detectors used in the NRF experiments at $\mathrm{HI} \gamma \mathrm{S}$.

an outstanding tool for measuring dipole transitions. The main advantage of this method is that both the excitation and the de-excitation processes proceed via the electromagnetic interaction, the best understood interaction in physics. Using this approach, the quality of the HI $\gamma \mathrm{S} \gamma$-ray beams provides tremendous advantages over bremsstrahlung beams. For example, the monoenergetic $\gamma$ ray beam allows excitation of only the desired levels of interest. In addition the parity of the excited state can be determined by measuring the angular distribution of the scattered photons with respect to the incoming unpolarized photon beam. For the case of even-even nuclei with ground state spin $J=0$ it is sufficient to measure the scattered radiation at two different azimuthal angles. The NRF setup at $\mathrm{HI} \gamma \mathrm{S}$ consist of four $60 \%$ HPGe detectors, positioned at $90^{\circ}$ to the beam axis, two in the horizontal and two in the vertical plane. An example of the experimental set-up used in the NRF measurements in shown in Fig. 1.

A $\gamma$-ray spectrum measured at zero degree with a 123\% HPGe detector is shown in Fig.2. The detector was placed in the beam operated in a low flux mode. A Monte-Carlo simulated response function of the detector is presented in red. A deconvolution analysis provides the FWHM of the photoabsorption peak displayed by the hatched area. The analysis of this peak shows that the HI $\gamma \mathrm{S}$ beam has an asymmetric Gaussian shape $\left(\mathrm{E}_{\gamma}=8.5_{-0.086}^{+0.064} \mathrm{MeV}\right)$ with total width of $\Delta \mathrm{E}_{\gamma}=140 \mathrm{keV}$ at $8.5 \mathrm{MeV}$. The average $\gamma$-ray flux on the target position using a $1.27 \mathrm{~cm}$ diameter lead collimator exceeds $1 \times 10^{6} \gamma / \mathrm{s}$ at $15 \mathrm{MeV}$ [13]. The flux of $\gamma$-rays can be increased in principle by increasing the current in the bunches in the storage ring and/or by operating with more than two electron bunches.

The scattering target consisted of $\mathrm{BaCO}_{3}$ powder of natural isotopic abundance packed into a lucite container $2.0 \mathrm{~cm}$ in diameter by $2.0 \mathrm{~cm}$ in hight.

The intensity distribution function derived within the angular correlation formalism [14] of a $0^{+} \stackrel{\vec{\gamma}}{\rightarrow} 1^{\pi} \stackrel{\gamma}{\rightarrow} 0^{+}$photon scattering cascade is given by

$$
W(\theta, \phi)=1+\frac{1}{4}\left[P_{2}(\cos \theta)-\frac{1}{2} \pi \cos (2 \phi) P_{2}^{(2)}(\cos \theta)\right],
$$




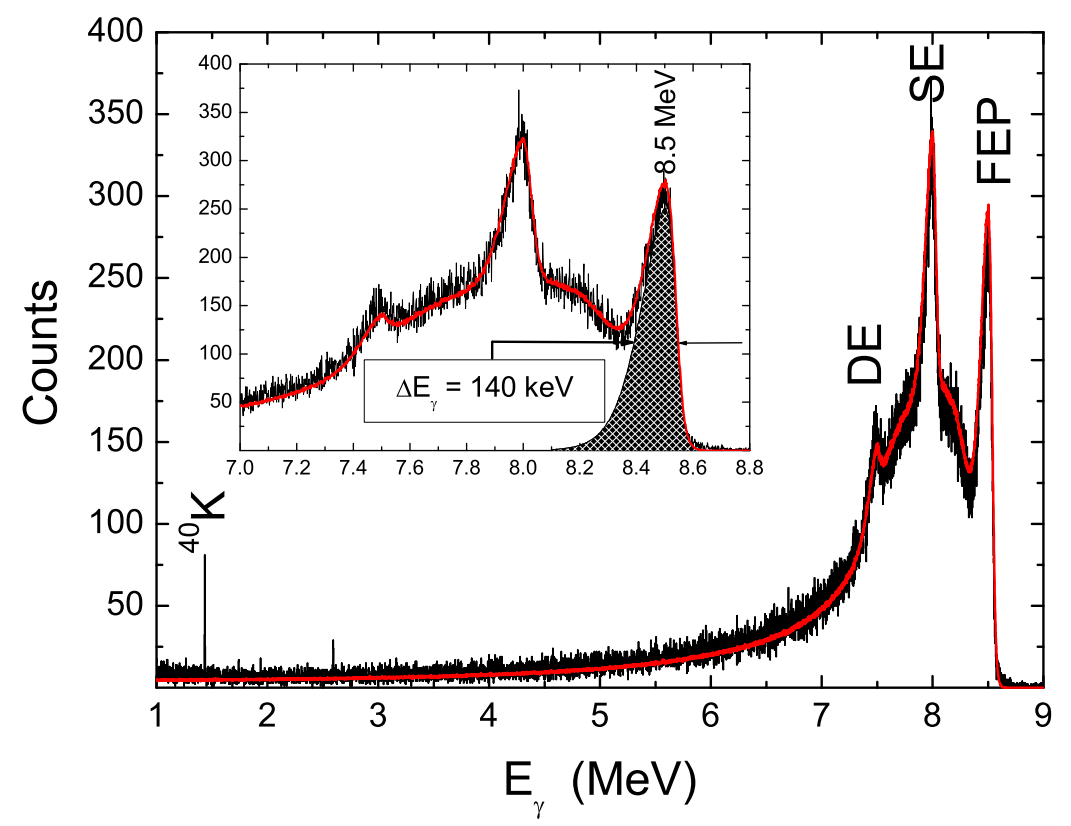

Figure 2: Gamma-ray spectrum measured with $123 \%$ HPGe detector at $0^{0}$ after $1.27 \mathrm{~cm}$ lead collimator. The full-energy peak (FEP), the single escape (SE), and double escape (DE) peaks are labeled. Imbeded figure shows the energy resolution of the FEP at $\mathrm{E}_{\gamma}=8.5 \mathrm{MeV}$ obtained from MCNPX simulation (red line)

with $P_{2}^{(2)}$ being the unnormalized associated Legendre polynomial of second order. Therefore, the theoretical intensity asymmetry is given by:

$$
\begin{aligned}
\Sigma_{t h}= & \frac{W\left(\phi=0^{\circ}\right)-W\left(\phi=90^{\circ}\right)}{W\left(\phi=0^{\circ}\right)+W\left(\phi=90^{\circ}\right)} \\
& =\pi=\left\{\begin{array}{cc}
+1 & J^{\pi}=1^{+} \\
\text {for } & \\
-1 & J^{\pi}=1^{-}
\end{array}\right.
\end{aligned}
$$

In the case of an $E 2$ excitation for a $0^{+} \rightarrow 2^{+} \rightarrow 0^{+}$cascade, the asymmetry is $\Sigma_{t h}(E 2)=-0.1$.

The experimental asymmetry is

$$
\Sigma_{e x}=\frac{A\left(\phi=0^{\circ}\right)-r\left(E_{\gamma}\right) A\left(\phi=90^{\circ}\right)}{A\left(\phi=0^{\circ}\right)+r\left(E_{\gamma}\right) A\left(\phi=90^{\circ}\right)}
$$

where $A(\phi)$ being the peak area in the NRF spectra obtained at $\phi=0^{\circ}, 90^{\circ}$; and $r\left(E_{\gamma}\right)$ the measured relative efficiency function. Parity quantum numbers of dipole excitations can then be assigned from azimuthal intensity asymmetry measured by our detector setup.

\section{Experimental Results}

Fig. 4 shows the experimental asymmetry of 13 dipole states in ${ }^{138} \mathrm{Ba}$ in the energy range from 


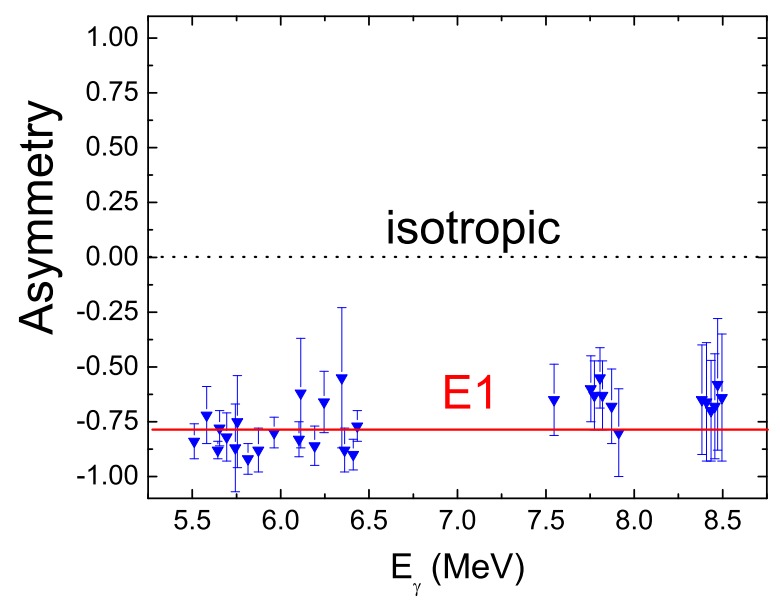

Figure 3: Asymmetry of the low-lying dipole states in ${ }^{138} \mathrm{Ba}$ using the $100 \%$ linearly polarized $\gamma$-ray beam at $\mathrm{HI} \gamma \mathrm{S}$.

7.5 to $8.5 \mathrm{MeV}$. All the dipole states deexcite via E1 transitions, i.e. the levels are $J^{\pi}=1^{-}$. Earlier NRF measurements at $\mathrm{HI} \gamma \mathrm{S}$ unambiguously showed that all of the dipole states in the energy region from 5.5 to $6.5 \mathrm{MeV}$ are also $J^{\pi}=1^{-}$[15]. Fig.5 shows the low-energy part (top panel) and the high energy part (bottom panel) of $\gamma$-ray spectra measured with the vertical (left) and horizontal (right) detectors relative to the polarization plane at $\mathrm{E}_{\gamma}=8.5 \pm 0.075 \mathrm{MeV}$. There are only a few dipole states which decay to the ground state (bottom-left panel). These states are observed only in the vertical detectors located in the plane perpendicular to the polarization plane. According to the azimuthal distribution these states exhibit E1 character. However, strong E2 transitions from the lowest $\left(J^{\pi}=2^{+}\right)$excited states in ${ }^{138} \mathrm{Ba}$ at $1435.8,2217.9$, and $2639.5 \mathrm{keV}$ to the ground state have been observed with the advantage of the pulsed $\gamma$-ray beam at the HI $\gamma \mathrm{S}$ facility (top panel). This experimental information is usually difficult to obtain in bremsstrahlung experiments due to the increasing nonresonant scattering at low-energies and the DC beam from the electron linear accelerator [3]. Note the logarithmic scale on the top panel and the absence of non beam related lines in the low-energy region.

The first question arises, how these low-lying $2^{+}$states were populated with $\gamma$-beam at 8.5 $\mathrm{MeV}$ and total width of $140 \mathrm{keV}$ ? It has to be mentioned that the decay intensity from the first $2_{1}^{+}$ state, for example, is 4 times higher compared to the ground state decay of the $8.43 \mathrm{MeV}$ level. There are three possible contributions to the population of the $2^{+}$states. The first one is that the monoenergetic beam at the HI $\gamma \mathrm{S}$ facility might have a low-energy tail. Such a tail could result from bremsstrahlung radiation of the relativistic electrons in the storage ring or from off-axis $\gamma$-rays entering into the $1.27 \mathrm{~cm}$ diameter $\mathrm{Pb}$ collimator. However, direct measurements of the $\gamma$-beam with low flux show that the intensity at $8.5 \mathrm{MeV}$, for example, is 40 times higher than in the $2 \mathrm{MeV}$ region (see Fig.2). Hence, this potential low-energy tail contribution should be negligible in the present experiment. The second possible contribution could be from neutrons produced in the lead 


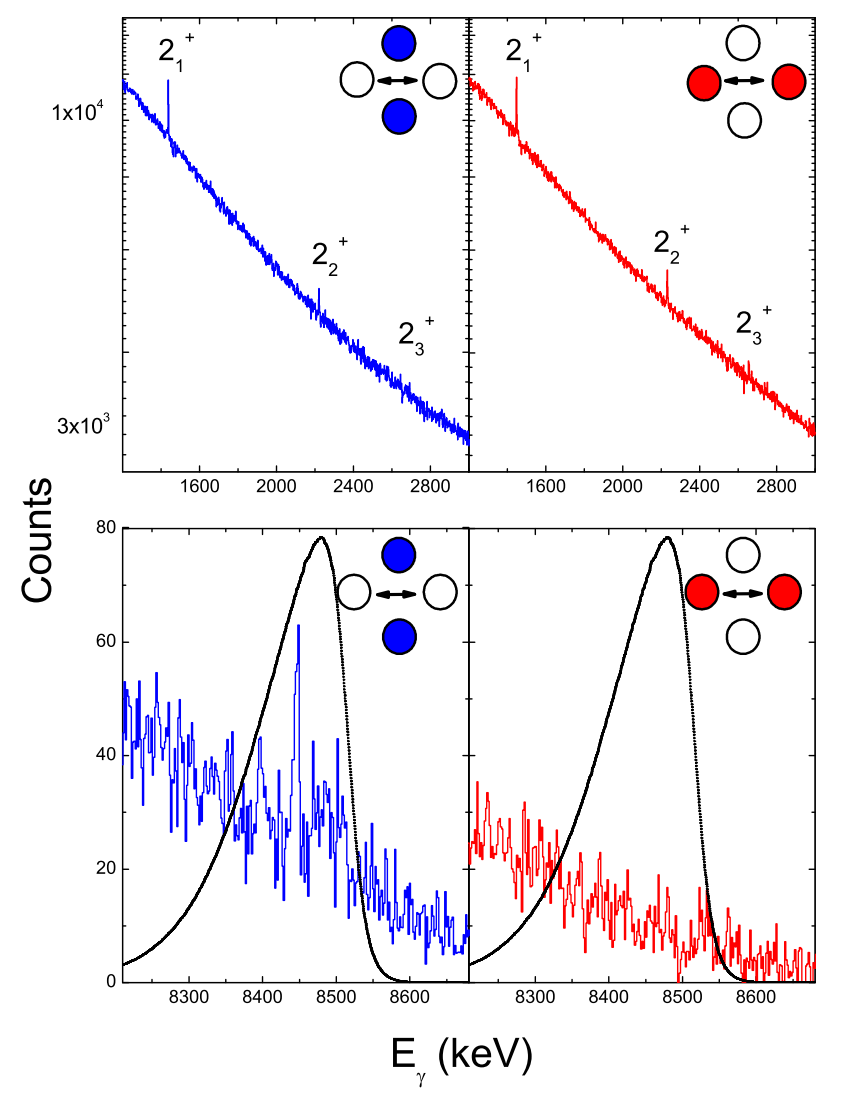

Figure 4: Low-energy part (top panel) and high-energy part (bottom panel) of the $\gamma$-ray spectra in the vertical (left) and horizontal (right) detectors at $\mathrm{E}_{\gamma}=8.5 \pm 0.075 \mathrm{MeV}$. The energy distribution of the $\gamma$-ray beam (bottom panel) is also shown.

collimator or neutrons produced in the DFELL storage ring. However, this potential contribution is removed by the pulsed technique used in the present experiment. The third possibility is for the primary beam of $\mathrm{E}_{\gamma}=8.5 \mathrm{MeV}$ to exhibit multiple Compton scattering in the barium sample itself. These multipole scattered photons can populate directly the $2^{+}$states. This possibility was simulated by Monte-Carlo methods and a negligible $\left(10^{-6}\right)$ contribution was obtained.

There are other important consequences of these measurements. As can be seen from Fig.4, the decays from the first three $J^{\pi}=2^{+}$states to the ground state is observed in both the horizontal and vertical detectors. The intensities of these transitions are almost identical for the horizontal and vertical detectors, indicating a loss of initial polarization. This observation is further supported by the lack of discrete inelastic transitions from the resonance energy to the $2^{+}$states. In Fig.5 the part of the spectrum taken with the vertical detectors is shown where a possible decay to the $2^{+}$ state should be visible. If indeed a branching were observed, such a line should be clearly visible above the hatched statistical background. However, no such peak was observed. This measurement was followed with a measurement at the lower beam energy of $\mathrm{E}_{\gamma}=8.2 \pm 0.07 \mathrm{MeV}$, where no resonance transitions were observed at the Darmstadt facility. No direct transitions were measured 


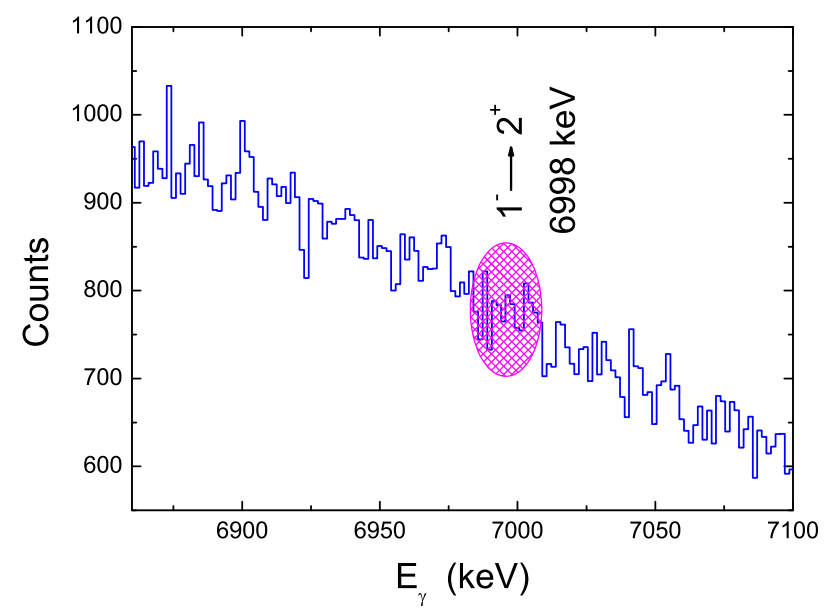

Figure 5: NRF spectrum of ${ }^{138} \mathrm{Ba}$ between 6850 and $7100 \mathrm{keV} .6988 \mathrm{keV}$ line indicate the region where the primary $\gamma$-transition from the $8.433 \mathrm{MeV}$ state to the $2_{1}^{+}$states should appear.

at the HI $\gamma \mathrm{S}$ facility either to the ground state or the $J^{\pi}=2^{+}$states. However, the low-energy $J^{\pi}=$ $2^{+}$state to ground state transitions still remain in both horizontal and vertical detectors. From this picture one can see that at $\mathrm{E}_{\gamma}=8.5 \pm 0.075 \mathrm{MeV}$, there are only a couple of dipole states strongly connected with the ground state. According to the statistical model there should be 430 dipole states $\left(J^{\pi}=1^{+}\right.$or $\left.1^{-}\right)$in the $140 \mathrm{keV}$ energy range. Therefore, there are a lot of unobserved dipole transitions and respectively $\gamma$-strength, which are hidden in the background of the previous nuclear resonance experiments. The advent of pulsed and monoenergetic HI $\gamma \mathrm{S}$ beam allows for accounting of the hidden $\gamma$-strength in ${ }^{138} \mathrm{Ba}$ at this energy. Our experiments at excitation energies above the neutron threshold at $8.8 \pm 0.08,9.1 \pm 0.08,9.5 \pm 0.08$, and $10.0 \pm 0.085 \mathrm{MeV}$, for example, did not identify any dipole transitions to the ground state or to any of the low-lying excited states in ${ }^{138} \mathrm{Ba}$.

\section{Discussion}

Microscopic calculations for the dipole-strength distribution were performed for ${ }^{138} \mathrm{Ba}$ within the framework of the quasiparticle-phonon model (QPM) [16]. The QPM results shows that the structure of the states contain a large neutron contribution (more than 90\%). These states correspond mainly to oscillations of weekly bound neutrons from $s$ - and $p$-shells. Microscopic study of the $1^{-}$states close to the particle threshold revealed that their structure is mostly an admixture of complex configurations. As a result, direct transitions from these states to the ground state are strongly hindered. Only a few dipole states in this region decay by strong E1 transitions to the ground state due to the PDR or GDR component in the structure of the state vector. Recent analysis of transition densities [10] showed that these excitations are related to the neutron PDR mode. The single phonon component in these dipole states, which is responsible for direct transitions to the ground state, is only a few percent. The analysis on dipole transition densities for different excitation energy regions in ${ }^{138} \mathrm{Ba}$ is presented in Fig. 6. 



Figure 6: One-phonon neutron and proton transition densities in ${ }^{138} \mathrm{Ba}$.

As discussed in previous sections, a certain concentration of electric dipole strength was found around $6.5 \mathrm{MeV}$ in $\mathrm{N}=82$ nuclei. The E1 strength distribution, taken from Ref. [9], is shown in Fig.7. The lowest-lying excitation in all $\mathrm{N}=82$ isotones is the two-phonon state $\left(2^{+} \otimes 3^{-}\right)$which determines the low-energy border of the PDR. The high-energy end is governed by the proton or neutron separation energy. The authors conclude that the centroid energy of the higher-lying dipole excitations is shifted to lower energies for more proton-rich nuclei. In addition, it was measured that the integrated total strength in ${ }^{144} \mathrm{Sm}$ decreases by a factor of 4 in comparison to the strength in ${ }^{138} \mathrm{Ba}$. To understand this systematic behavior of the E1 strength distribution, statistical and QPM models were invoked. The dipole strength in Fig.7 is observed to damp out as the excitation energy approaches either the neutron $\left(\mathrm{B}_{n}\right)$ or proton $\left(\mathrm{B}_{p}\right)$ binding energy. Q-value systematics for the $\mathrm{N}=82$ nuclei show that $\mathrm{B}_{n}$ increases with increasing proton number, while $\mathrm{B}_{p}$ goes in the opposite direction. The different Q-values will provide ${ }^{142} \mathrm{Nd}$ and ${ }^{144} \mathrm{Sm}$ nuclei with less energy space in comparison to ${ }^{138} \mathrm{Ba}$, for example. Hence the total E1 strength will be in favour of high-Q value nuclei. The observed centroid shift in Ref. [9] is most probably due to the Q-value systematics.

Another important question that remains to be answered is how this low-energy collective PDR mode evolves to the GDR mode. Fig.8(a) presents the photoneutron cross sections for the stable $\mathrm{N}=82$ nuclei in the GDR region [17]. The photoneutron cross sections shows almost identical width, maximum, and energy of the giant resonance as one adds protons. Fig.8(b) shows the total absorption cross section below the particle separation energy using the statistical model. All these calculations have the same total absorption value in the PDR region based on the statistical model. Hence it will be very interesting to measure if the direct transitions in ${ }^{142} \mathrm{Nd}$ or ${ }^{144} \mathrm{Sm}$ will be accompanied with an even stronger statistical decay. Figure 9(a) shows the constant temperature (CT) and back-shifted Fermi gas model (BSFG) obtained as fit [19] to the experimental cumulative number of dipole levels in ${ }^{138} \mathrm{Ba}$ measured so far. This heavy mass nucleus has one of the most extensively studied level scheme up to $8.5 \mathrm{MeV}$ excitation energy. Above $4.5 \mathrm{MeV}$ the predicted level densities [19] increasingly surpass the number of measured dipole states. Apparently the 


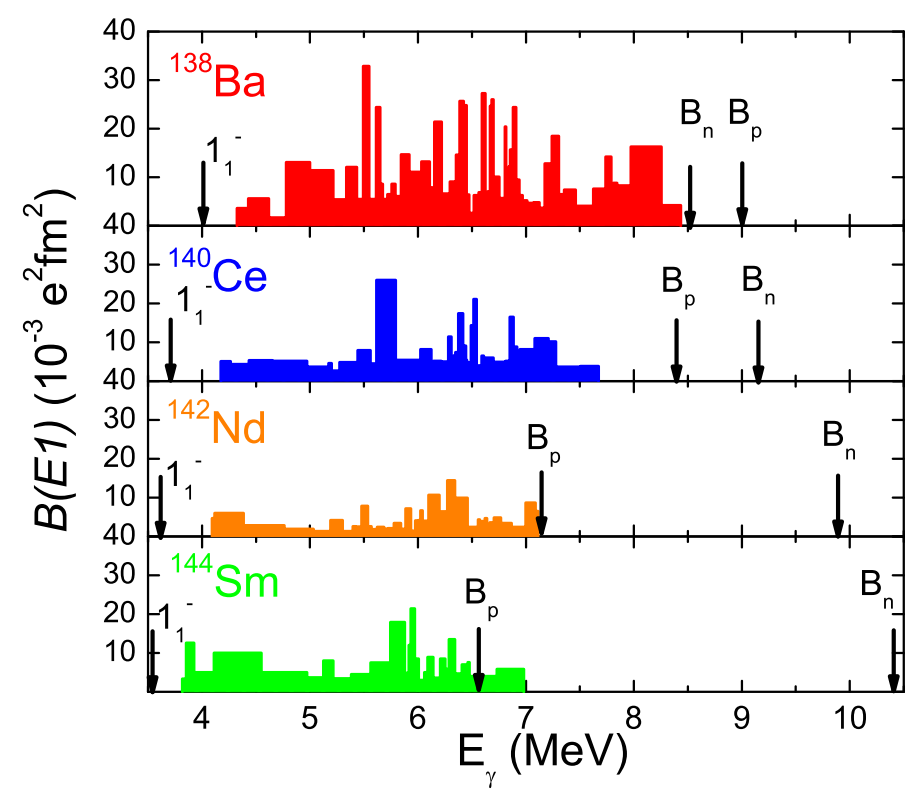

Figure 7: $\mathrm{B}(\mathrm{E} 1)$ strength distribution in the $\mathrm{N}=82$ isotones. Data is taken from [17].

Table 1: One-phonon $1^{-}$states below the neutron separation energy for $\mathrm{N}=82$ nuclei. Only the largest neutron (' $v$ ') and proton (' $\pi$ ') components are given.

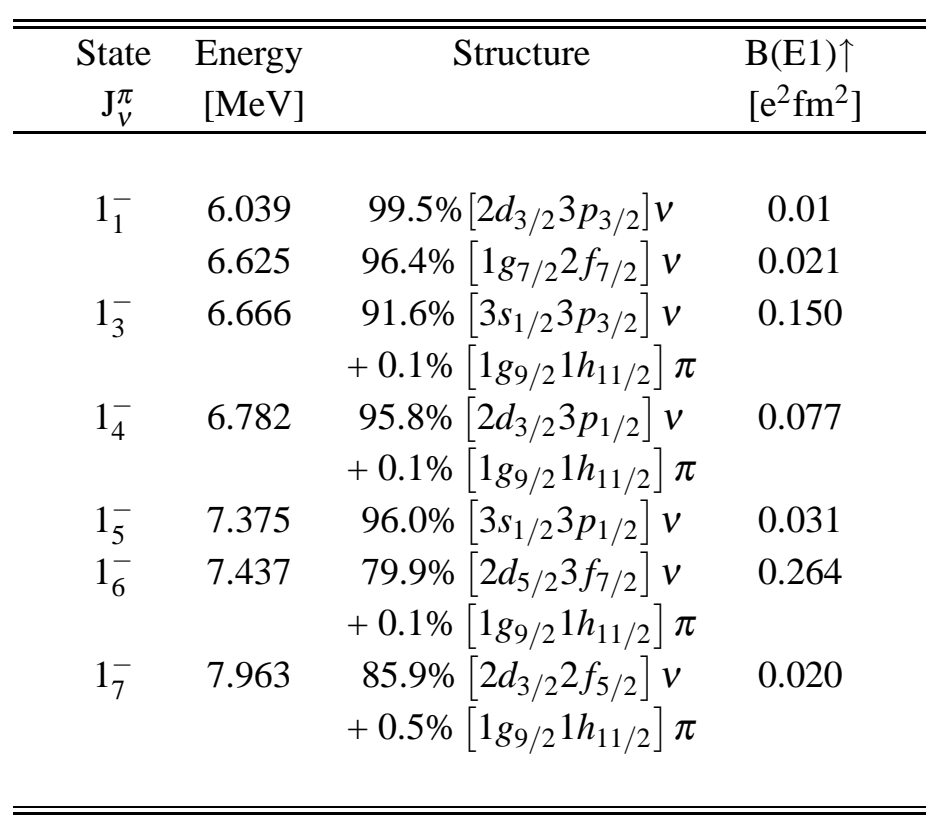




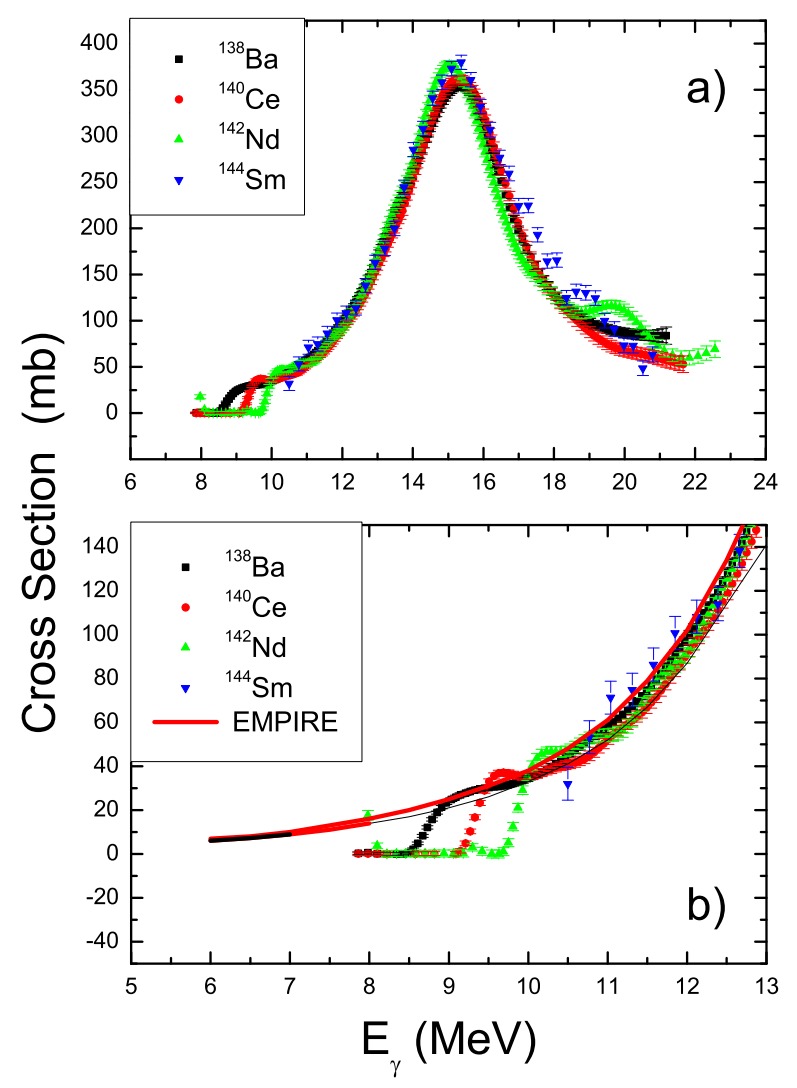

Figure 8: Top part:photoneutron absorption cross section for $\mathrm{N}=82$ nuclei in the GDR region. Bottom part: low-energy tail of the GDR extended by statistical calculations below the particle separation energy.

NRF experiments start missing a lot of levels at $\mathrm{E} \geq 4.5 \mathrm{MeV}$. Since the levels close to the neutron threshold produce many but weak branching or cascade transitions, a large portion of the strength of these states is likely to be missed experimentally. The intensity of the branching transitions is much smaller, $\approx 100$, than the corresponding ground-states transition and they do not produce identifiable photopeaks in the measured spectrum. However, the fact that there are so many, at the end they will "rain" down by fast E1 or M1 transitions to the low-energy $2^{+}$states. Statistical calculations show that its takes 3-4 $\gamma$-ray transitions to release the initial excitation energy. In principle, also M1-transitions contribute to the dipole strength, but the average M1 strength is typically 1-2 orders of magnitude smaller compared to the E1 strength, and it is usually not taken into account.

The photoabsorption cross section below the particle emission threshold can be writen as:

$$
\sigma_{t}=\sigma_{e l}+\sigma_{\text {inel }}
$$

where $\sigma_{e l}$ is the cross section due to all $\gamma$-ray transitions decaying directly into the ground state, while $\sigma_{\text {inel }}$ is the cross section due to the $\gamma$-ray transitions decaying indirectly to the ground state. 




Figure 9: Calculated cumulative number of levels (continous curve) of ${ }^{138} \mathrm{Ba}$ with spin $1^{+}$or $1^{-}$with the CT and BSFG model formula [15]. Measured dipole levels are shown with triangles $[9,15,18]$.

Unfortunately, in the present experiment the absolute cross section was not able to be determine due to the un-precisely known mass of the Ba target. Hence, only a relative intensity distribution between the elastic and inelastic part can be extracted.

Table 2 shows the relative intensity distribution of the first three low-lying $2^{+}$states to the ground state, also all elastic transitions from $\mathrm{E}_{\gamma}=8.5 \pm 0.075 \mathrm{MeV}$. These transitions are corrected to the detector efficiency, branching ratio and $\gamma$-ray attenuation in the barium sample. As it was discussed earlier, primary $\gamma$-ray transitions from the energy range of $8.5 \pm 0.075 \mathrm{MeV}$ to the first $2^{+}$excited states or any other excited states were not observed. The present experimental intensity distribution is confirmed by the statistical calculations. The second row of Table 2 shows the EMPIRE [20] calculations for the first three low-lying $2^{+}$states to the ground state, and the elastic transitions from $\mathrm{E}_{\gamma}=8.5 \pm 0.075 \mathrm{MeV}$ normalized to the transitions from the $2^{+}$state to the ground state. The third row shows the absolute photoabsorption cross sections calculated by EMPIRE code. Different models of E1 $\gamma$-ray strength functions, proposed in RIPL-2 [21], were used in the present calculations. However, the values for the partial cross sections did not change. This result is due to the fact that at excitation energies close to the neutron separation energy the photoabsorption cross section is insensitive to the choice of the $\gamma$-ray strength function.

It should be noted that there are states which decay will bypass the low-lying states. These bypass transitions are usually steaming from the intermediate $(\mathrm{E}=3-4 \mathrm{MeV})$ or continuum energy region $(\mathrm{E}=4-9 \mathrm{MeV})$ and have a strength below the experimental detector limit. However, the missing strength can be estimate by the statistical model. For example, EMPIRE calculation predicted $\sigma_{t}=19 \mathrm{mb}$ at $\mathrm{E}_{\gamma}=8.5 \mathrm{MeV}$, which makes the ratio of inelastic versus direct transitions $=7.9$. 
Table 2: Relative intensity distribution in ${ }^{138} \mathrm{Ba}$ at $\mathrm{E}_{\gamma}=8.50(14) \mathrm{MeV}$.

\begin{tabular}{ccccc}
\hline \hline & $2_{1}^{+} \rightarrow$ g.s. & $2_{2}^{+} \rightarrow$ g.s. & $2_{3}^{+} \rightarrow$ g.s. & $8.50(14) \mathrm{MeV} \rightarrow$ g.s. \\
& & & & \\
\hline Experiment & $55.0(30) \%$ & $8.7(20) \%$ & $4.5(19) \%$ & $31.8(23) \%$ \\
& & & & \\
\hline EMPIRE [20] & $56 \%$ & $10 \%$ & $4 \%$ & $30 \%$ \\
& $2.8 \mathrm{mb}$ & $0.5 \mathrm{mb}$ & $0.2 \mathrm{mb}$ & $1.5 \mathrm{mb}$ \\
\hline \hline
\end{tabular}

\section{Summary}

In summary, the systematic parity measurements on ${ }^{138} \mathrm{Ba}$ at $\mathrm{HI} \gamma \mathrm{S}$ verified for the first time that the observed dipole strength below the particle threshold is predominantly electric dipole. Our findings are in agreement with other QPM predictions for the character of this dipole mode of excitation. The high level density at the particle separation energy leads to many weak transitions which are difficult to observe directly. In addition these weak transitions decay via cascades to many intermediate levels. Therefore much of the information on dipole strength is missing from observation of deexcitations to the ground state only. These transitions are so weak but numerous that their superposition can be observed as a continuum of unresolved strength. Despite the fact that the PDR exhibits well pronounce collective structure below the neutron separation energy the inelastic transitions will mostly dictate the reaction rate in this energy region. The present measurements also show that the monoenergetic and pulsed beam from the HI $\gamma \mathrm{S}$ facility opens up a new opportunity for direct measurements of the level density and the radiative $\gamma$-ray strength functions by measuring not only the direct gamma transitions but also the non-resonance part of the decay process.

We thank the technical staff at the DFELL for providing excellent $\gamma$-ray beams. We are also grateful to G.E. Mitchell, M. Krticka, and G. Rusev for fruitful discussion. This work was partially supported by the Office of Science of the US Department of Energy DE-FG02-97ER41033, Grant Nos. DE-FG02-97ER41033, DE-FG02-97ER41042, and DE-FG02-97ER41041.

\section{References}

[1] F.R. Metzger, Prog. in Nucl. Phys. 7, 54 (1959).

[2] U.E.P. Berg and U. Kneissl, Ann. Rev. Nucl. Part. Sci. 37, 33 (1987).

[3] U. Kneissl et al., Prog. Part. Nucl. Phys. 37, 349 (1996).

[4] U. Kneissl et al., J. Phys. G: Nucl. Part. Phys. 32 R217 (2006).

[5] A. Richter, Prog. Part. Nucl. Phys. 13, 1 (1985).

[6] C. Fransen et al., Phys. Rev. C 70, 044317 (2004).

[7] D. Savran et al., Phys. Rev. C 71, 034304 (2005).

[8] A. Zilges at al., Phys. Lett. B 542, 43 (2002). 
[9] S. Voltz et al., Nucl. Phys. A 779,1 (2006).

[10] N. Tsoneva et al., Phys. Lett. B 586, 213 (2004).

[11] M. Arnould, S. Goriely., Phys. Rep. 384, 1 (2003).

[12] V. Litvinenko et al., Phys. Rev. Lett. 78, 4569 (1997).

[13] A.P. Tonchev et al., Nucl. Instrum. Methods Phys. Res. B 241, 170 (2005).

[14] L.W. Fagg and S.S. Hanna, Rev. Mod. Phys. 31, 711 (1959).

[15] N. Pietralla et al., Phys. Rev. Lett 88, 012502 (2002).

[16] V.G. Soloviev, Theory of Atomic Nuclei: Quasiparticles and Photons (Institute of Physics, Bristol, 1992).

[17] S.S. Dietrich and B.L. Berman, "Atlas of photonuclear cross-sections obtained with monoenergetic photons", Atomic Data and Nuclear Data Tables. 38, 199 (1988).

[18] R.-D. Herzberg at al., Nucl. Phys. A 592, 211 (1995).

[19] T. von Egidy and D. Bucurescu, Phys. Rev. C72, 044311 (2005).

[20] EMPIRE:Nuclear reaction model code. http://www.nndc.bnl.gov/empire219/.

[21] IAEA-CRP, Reference Input Parameter Library, Phase II (RIPL-2), 2002. 